\title{
A revista Perspectivas em Gestão \& Conhecimento (PG\&C): Criação, gestão editorial e espectro de temas publicados
}

\author{
Luciana Ferreira da Costa \\ Emeide Nóbrega Duarte \\ Jorge de Oliveira Gomes \\ Universidade Federal da Paraíba - UFPB, Brasil \\ Alan Curcino Pedreira da Silva \\ Universidade Federal de Alagoas - UFAL, Brasil
}

COMMUNICATIONS

\begin{abstract}
Resumo
Objetivo. Apresenta o espectro de temas publicados na revista Perspectivas em Gestão \& Conhecimento (PG\&C) identificados por meio da frequência de ocorrência das palavras-chave dos artigos.

Método. Utiliza análise categorial temática. Assim, foram determinadas como categorias de trabalho os itens de caracterização da revista quanto aos seus dados gerais, processo de gestão editorial e os principais assuntos dos artigos publicados extraídos das palavras-chave, numa abordagem qualitativa-narrativa com aporte de dados quantitativos.

Resultados. Evidencia os assuntos com maior índice de freqüência: Gestão do conhecimento, Gestão da informação, Ciência da Informação, Aprendizagem, Sistemas de informação e Tecnologias de informação. Este resultado expressa a tendência dos estudos convergentes entre a Administração e a Ciência da Informação.

Conclusões. Conclui que o periódico PG\&C já se firmou como veículo de referência nacional para diversas áreas que tratam da Gestão e do Conhecimento como objeto inter-relacional de estudo, não deixando de reconhecer aí a visibilidade e interesse internacional.
\end{abstract}

Palavras-chave

Periódico científico ; Perspectivas em Gestão \& Conhecimento (PG\&C) ; Gestão editorial ; Administração ; Ciência da Informação

The journal Perspectivas em Gestão \& Conhecimento (PG\&C): Creation, editorial management and spectrum of topics published

\begin{abstract}
Objective. Presents the spectrum of topics published in the scientific journal "Perspectivas em Gestão \& Conhecimento" (PG\&C) identified by the frequency of occurrence of keywords in the articles.

Method. The study used thematic category analysis. So were determined as job categories the journal characterization of items for your general data, editorial management process and the main subjects of the articles taken from the keywords, a qualitativenarrative approach, with quantitative data input

Results. This results express the trend of converging studies between the Administration and Information Science.

Conclusions. Concludes that the scientific journal PG\&C has established itself as a vehicle for national reference for various areas dealing with the management and knowledge as inter-relational object of study, not failing to recognize the visibility and international interest.
\end{abstract}

Keywords

Scientific journal ; Perspectivas em Gestão \& Conhecimento (PG\&C) ; Editorial Management ; Management ; Information Science 


\section{Trajetória de criação da revista}

A revista Perspectiva em Gestão \& Conhecimento (PG\&C) nasceu de iniciativa da Coordenação do Curso de Graduação em Administração (Gestão 2009-2011) do Centro de Ciências Sociais Aplicadas da Universidade Federal da Paraíba (UFPB), em conjunto com a experiência de ensino da disciplina Metodologia do Trabalho Científico (MTC), ministrada pela primeira autora no Curso de Graduação em Administração (semestre letivo 2010.2 disciplina ofertada pelo Departamento de Ciência da Informação). No empreender da PG\&C houve, ainda, a cooperação entre docentes pesquisadores vinculados ao Departamento de Administração, ao Departamento de Ciência da Informação e ao Departamento de Filosofia da UFPB, contando com o apoio técnico-científico do Instituto Brasileiro de Informação em Ciência e Tecnologia (IBICT). A este empreendimento cooperativo, somam-se, pesquisadores nacionais e estrangeiros como membros da Equipe Editorial ou como referees ad hoc convidados.

Inicialmente, a ideia apoiada em perspectiva interdisciplinar, parte da área da Ciência da Informação no elo com a área da Administração, o que se estendeu de forma prática e epistemologicamente pela missão proposta da revista e pela demanda de submissões de origens diversas - sob processo de recebimento de submissões em fluxo contínuo até segunda ordem por decisão editorial -, leitores de várias origens e uma equipe editorial (pesquisadores atuantes e comprometidos com sua área) que atendesse a toda essa demanda.

Ratificando o exposto quanto às áreas, segundo Oliveira, Pinheiro e Andrade (2011), recentemente, a Administração tem se aproximado da Ciência da Informação em função da atual realidade informacional na perspectiva de aprofundar os estudos interdisciplinares. Os autores destacam que a preocupação atual com a Gestão do conhecimento e com a Inteligência competitiva tem contribuído bastante para esse intercâmbio.

Nessa perspectiva, a revista PG\&C tem como missão publicar trabalhos originais e inéditos relacionados com as temáticas Gestão e Conhecimento sob abordagens que priorizem os diálogos disciplinares (inter/pluri/multi/trans) e que se configurem como contribuições para a construção e o desenvolvimento de novos conhecimentos (PG\&C, 2013a).

Desde a idealização até a divulgação da PG\&C, testemunhou-se a predisposição e participação de uma rede acadêmico-profissional. Desta rede, além daquelas instituições já mencionadas, destaca-se a Coordenação de Aperfeiçoamento de Pessoal de Nível Superior (CAPES), a Sociedade Brasileira para o Progresso da Ciência (SBPC), a Associação Nacional de Pesquisa e Pós-Graduação em Administração (ANPAD), a Associação Nacional de Pesquisa e Pós-Graduação em Ciência da Informação (ANCIB), a Sociedade Brasileira de Gestão do Conhecimento (SBGC), coordenações de programas de pós-graduação nacionais e estrangeiros, dentre outras instituições e pessoas que divulgaram/replicaram mensagens em seus sites e redes sociais (blogs, twitter, orkut, etc.) e, muitas vezes, direcionaram solicitações de informação e sugestões para os e-mails dos membros da Equipe Editorial. Como consequência, as estatísticas de visitas ao site do periódico apontaram, até o mês de maio de 2011 - antes do lançamento do primeiro número -, acessos originados de 22 países $^{1}$ (GOMES; COSTA, 2011).

Após a publicação do primeiro número, em junho de 2011, o feedback dessa rede de inteligência coletiva confirmou a demanda por um canal de comunicação científica com especificidade proposta pela PG\&C.

Pelo panorama exposto, este relato descreve a experiência do processo editorial de um recente canal brasileiro de comunicação científica, a revista eletrônica e de acesso aberto intitulada Perspectivas em Gestão \& Conhecimento (PG\&C).

Como estratégia de construção deste relato, utilizou-se da análise categorial temática - "contagem de um ou vários temas ou itens de significação, numa unidade de codificação previamente determinada” - (BARDIN, 2008, p. 73). Assim, determinaram-se como categorias de trabalho os itens de caracterização da revista quanto aos seus dados gerais, processo de gestão editorial e os principais assuntos dos artigos publicados extraídos das palavras-chave dos artigos, numa abordagem qualitativa-narrativa, com aporte de dados quantitativos (RICHARDSON, 1999). A seguir, apresenta-se a descrição da PG\&C como experiência bem-sucedida na criação e manutenção de uma revista científica. 


\section{A revista Perspectivas em Gestão \& Conhecimento (PG\&C)}

Nesta seção, apresentam-se os dados gerais da revista, resultados do processo de gestão editorial e os principais assuntos dos artigos publicados.

\subsection{Dados gerais do periódico}

A PG\&C é uma revista científica eletrônica de acesso aberto de periodicidade semestral, que utiliza o software Open Journal System (OJS) - versão 2.X, baseado no Movimento de Acesso Aberto - sendo disponibilizada à comunidade científica nacional e internacional por meio do Portal de Periódicos Científicos Eletrônicos da UFPB, desde dezembro de 2010 (quando da disponibilização online do sistema da revista), através do seguinte endereço eletrônico: http://periodicos.ufpb.br/ojs2/index.php/pgc.

Quanto ao OJS, o software foi criado em 1998 pelo Public Knowledge Project (PKP) da University of British Columbia, Canadá, sob cooperação do Centro Canadense de Estudos em Editoração e da Biblioteca da Simon Fraser University, sendo uma solução de código livre para gerenciamento e publicação de revistas científicas na Internet, devendo, portanto, ser instalado em um servidor Web. Este sistema, além de gratuito, é altamente flexível, operado pelos próprios Editores de revistas científicas, responsáveis pela administração do processo de publicação, sendo recomendado por instituições governamentais brasileiras como a CAPES, o CNPq e o IBICT, este último tendo customizado uma versão em português amplamente difundida, o Sistema Eletrônico de Editoração de Revistas (SEER) (SILVA; COSTA, 2010).

Nessa perspectiva, segundo o PKP, o sistema visa:

reduzir o tempo e a energia devotados às tarefas administrativas e de secretariado associadas à produção de uma revista, enquanto melhora a preservação dos registros e a eficiência dos processos editoriais. Busca também aperfeiçoar a qualidade da publicação científica e acadêmica através de várias inovações, desde a transparência das políticas ao aprimoramento da indexação da revista (PKP; IBICT, 2009, p. 6).

Mais uma iniciativa do IBICT, com o apoio da Finep - Inovação e Pesquisa, surgiu em janeiro de 2013 com o intuito de salvaguarda do patrimônio científico, tecnológico e cultural, de forma distribuída, por instituições de pesquisa parceiras do IBICT (atualmente desenvolvida com cinco universidades). A iniciativa atende pelo nome de Rede Brasileira de Serviços de Preservação Digital ou Rede Cariniana, um serviço de preservação digital de documentos eletrônicos brasileiros com a finalidade de garantir o acesso contínuo a longo prazo dos conteúdos arquivados digitalmente. A PG\&C foi indicada pela Coordenação do Portal de Periódicos Científicos Eletrônicos da UFPB para ser preservada na Rede Cariniana, que possibilita que cada instituição armazene os dados fisicamente de forma interligada por meio do software LOCKSS ${ }^{2}$.

A PG\&C publica os seguintes tipos de documentos: editoriais, apresentações de seções ou de números, cartas aos leitores, artigos de revisão, relatos de pesquisa, relatos de experiência, memórias de eventos científico-profissionais, pontos de vista/comentários, entrevistas, resenhas e expedientes.

A revista utiliza o processo de avaliação de submissões de originais do tipo double blind peer review para artigos de revisão, relatos de pesquisa, relatos de experiência, memórias de eventos científico-profissionais e resenhas. Todos os originais publicados estão disponibilizados de acordo com a Licença "BY-NC-DC" do Creative Commons 3.0 Brasil (obrigatoriedade de atribuição de créditos/vedado uso comercial/vedada criação de obras derivadas/permitida citação referenciada), observando às suas normas, bem como, ainda, à Declaração de ética e boas práticas de publicação do periódico (PG\&C, 2013b).

A abrangência internacional e aceite de submissões nas línguas portuguesa, inglesa ou espanhola permitiu ao periódico PG\&C estar registrado/indexado em diferentes e respeitadas bases de dados e outros sistemas nacionais e internacionais conforme detalhado no Quadro 1. 


\section{Quadro 1 : Registro e indexação da PG\&C}

\begin{tabular}{|c|c|}
\hline Base de dado & Detalhe \\
\hline LATINDEX & $\begin{array}{l}\text { Sistema Regional de Información em Línea para Revistas Científicas de America Latina, el Caribe, } \\
\text { España y Portugal }\end{array}$ \\
\hline DOAJ & Directory of Open Acess Journal, Developed by Lund University Library \\
\hline DRJI & Directory of Research Journals Indexing, from Solapur - Maharashtra, India \\
\hline LISA & Library and Information Science Abstracts, developed by ProQuest Periodicals \\
\hline Dialnet & $\begin{array}{l}\text { Portal bibliográfico de acceso libre y gratuito, mantenido por la Fundación Dialnet y la Universidad de La } \\
\text { Rioja }\end{array}$ \\
\hline CAPES & $\begin{array}{l}\text { Portal de Periódicos da Coordenação de Aperfeiçoamento de Pessoal de Nível Superior - CAPES, } \\
\text { Ministério da Educação do Brasi }\end{array}$ \\
\hline NewJour & Electronic Journals \& Newsletter, developed by Georgetown University Library \\
\hline SFX & Knowledge Base - developed by Ex Libris Group Ltd \\
\hline TULIPS & developed by University of Tsukuba Library (筑波大学附属図書館の) \\
\hline CCUC & Catàleg Col·lectiu de les Universitats de Catalunya \\
\hline CB-UdG & Catàleg de la Biblioteca de la Universitat de Girona \\
\hline UNIVERSIA.NET & Biblioteca Universia de Recursos de Aprendizaje, mantenido con el mecenazgo del Grupo Santander \\
\hline Sumarios.org & $\begin{array}{l}\text { Sumários de Revistas Brasileiras, sob responsabilidade da Fundação de Pesquisas Científicas de } \\
\text { Ribeirão Preto }\end{array}$ \\
\hline SEER & $\begin{array}{l}\text { Portal do Sistema Eletrônico de Editoração de Revistas, sob responsabilidade do Instituto Brasileiro de } \\
\text { Informação em Ciência e Tecnologia do Ministério da Ciência, Tecnologia e Inovação do Brasil }\end{array}$ \\
\hline DIADORIM & $\begin{array}{l}\text { Diretório de Políticas de Acesso Aberto das Revistas Científicas Brasileiras, sob responsabilidade do } \\
\text { Instituto Brasileiro de Informação em Ciência e Tecnologia do Ministério da Ciência, Tecnologia e } \\
\text { Inovação do Brasil }\end{array}$ \\
\hline LivRe & $\begin{array}{l}\text { Portal para Periódicos de Livre Acesso na Internet, sob responsabilidade da Comissão Nacional de } \\
\text { Energia Nuclear do Ministério da Ciência, Tecnologia e Inovação do Brasil }\end{array}$ \\
\hline UFPB & Portal de Periódicos Científicos Eletrônicos da Universidade Federal da Paraíba \\
\hline Portal Spell & $\begin{array}{l}\text { Scientific Periodicals Electronic Library, desenvolvido pela Associação Nacional de Pós-Graduação e } \\
\text { Pesquisa em Administração e pelo Instituto Brasileiro de Estudos e Pesquisas Sociais }\end{array}$ \\
\hline Base PERI & $\begin{array}{l}\text { Periódicos Indexados pela Biblioteca Professora Etelvina Lima da Universidade Federal de Minas } \\
\text { Gerais }\end{array}$ \\
\hline BRAPCl & $\begin{array}{l}\text { Base de Dados Referencial de Artigos de Periódicos em Ciência da Informação da Universidade } \\
\text { Federal do Paraná }\end{array}$ \\
\hline InfoBCl & Divulgando informações, compartilhando conhecimentos (plataforma wordpress.com) \\
\hline
\end{tabular}

Fonte: PG\&C (2013c)

No mês de fevereiro de 2013, a PG\&C ingressou no sistema Qualis ${ }^{3}$ de Avaliação de Periódicos Científicos da CAPES, obtendo classificações nos seguintes estratos descritos no Quadro 2: 
Quadro 2 : Classificação da PG\&C no sistema Qualis

\begin{tabular}{|c|l|}
\hline Qualis & \\
\hline \multirow{2}{*}{ B1 } & Ciências Sociais Aplicadas I \\
\cline { 2 - 2 } & Arquitetura e Urbanismo \\
\cline { 2 - 3 } & Interdisciplinar \\
\hline \multirow{2}{*}{ B2 } & Educação \\
\cline { 2 - 3 } & Planejamento Urbano e Regional/Demografia \\
\hline \multirow{2}{*}{ B3 } & Engenharia III \\
\cline { 2 - 3 } & Administração, Ciências Contábeis e Turismo \\
\hline
\end{tabular}

Fonte: Capes (2013)

Até a realização deste relato entre dezembro de 2013 e fevereiro de 2014, a PG\&C contabilizava nove números publicados, conforme descrição constante do Quadro 3, atendendo rigorosamente sua periodicidade semestral. Consta deste total o lançamento de um número especial por ano sempre no mês de outubro, englobando temáticas de interesse contemporâneo, definidas pelo Comitê Editorial do periódico, editorado por pesquisador convidado expert na temática do número especial.

O primeiro número especial (v. 1 de 2011) se dedicou à temática "Arquitetura da Informação", o segundo número especial (v. 2 de 2012) se dedicou à temática "Gestão da Informação e do Conhecimento no âmbito da Ciência da Informação". O terceiro número especial (v. 3 de 2013) versou sobre a temática "Psicologia, Gestão e Conhecimento". No Quadro 3 descreve-se os volumes da PG\&C publicados até então, seguidos da quantidade de itens publicados no tocante aos Artigos de Revisão (AR), Relatos de Pesquisa (RP), Relatos de Experiência (RE), Memória de Evento Científico-Profissional (MECP), Resenhas (R), Carta ao Leitor (CL) e Pontos de Vista/Comentários (PVC):

Quadro 3 : Descrição dos volumes publicados da PG\&C

\begin{tabular}{|c|c|c|c|c|c|c|c|c|}
\hline ANO & ESPECIFICAÇÃO DA PG\&C & AR & RP & RE & MECP & $\mathbf{R}$ & CL & PVC \\
\hline \multirow{3}{*}{2011} & Volume 1, número 1 & 5 & 6 & 2 & - & - & - & - \\
\hline & $\begin{array}{l}\text { Volume 1, Número Especial - Perspectivas em } \\
\text { Arquitetura da Informação }\end{array}$ & 3 & 2 & 1 & 7 & 2 & - & - \\
\hline & Volume 1, número 2 & 8 & 8 & - & - & - & 1 & 1 \\
\hline \multirow{3}{*}{2012} & Volume 2, número 1 & 6 & 6 & 1 & - & - & - & - \\
\hline & $\begin{array}{l}\text { Volume 2, Número Especial - Gestão da Informação } \\
\text { e do Conhecimento no âmbito da Ciência da } \\
\text { Informação }\end{array}$ & - & - & - & 12 & - & - & 1 \\
\hline & Volume 2, número 2 & 6 & 5 & - & 1 & - & - & 1 \\
\hline \multirow{3}{*}{2013} & Volume 3, número 1 & 5 & 6 & 1 & - & 1 & - & - \\
\hline & $\begin{array}{l}\text { Volume 3, Número Especial - Psicologia, Gestão e } \\
\text { Conhecimento }\end{array}$ & 6 & 8 & - & - & - & - & - \\
\hline & Volume 3, número 2 & 5 & 13 & - & - & - & 1 & - \\
\hline \multicolumn{2}{|r|}{ Totais } & 44 & 54 & 5 & 20 & 3 & 2 & 3 \\
\hline
\end{tabular}


Fonte: PG\&C (2014)

Assim, tem-se que ao longo de sua idealização e evolução, a PG\&C prima pela regularidade, pelo compromisso com a qualidade dos artigos publicados e pelo rigor e ética editorial.

\subsection{Processo editorial}

PG\&C possui o completo processo de gestão editorial automatizado possibilitado pelo OJS, conforme apresentado na Figura 1.

Autor

\section{Submisstlo}

Autor envia o documento pelo sistema e alimenta metadados para indexação pelo protocolo OAI
Editor $\mathrm{e}$ Editor de Seçăo
Editor Gerente, Avaliador, Editor de Texto, Editor de Layout e Leitor de Provas

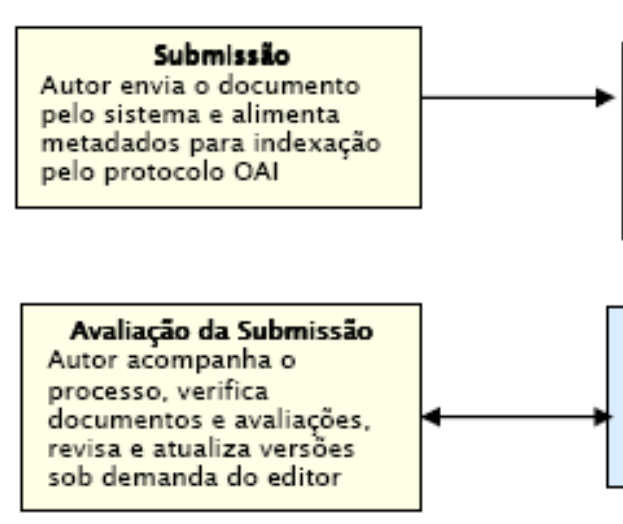

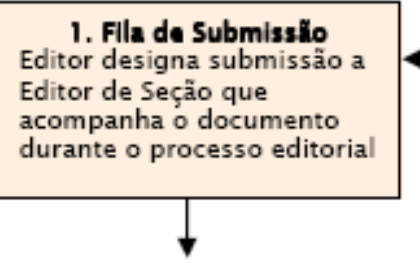

2. Avaliação da Submissäo

a) Verifica submissão

b) Gerencia avaliação

c) Toma decisão editorial
Ediçäo da Submissão Autor revisa edições e provas para publicação

\begin{tabular}{l} 
Editor Gerente \\
Editor \\
Editor de Seção \\
Editor de Texto \\
Editor de Layout \\
Leitor de Prova \\
Autor \\
Leitor \\
\hline $\begin{array}{l}\text { Open Journal Systems é } \\
\text { um sistema de código } \\
\text { livre do Projeto Public } \\
\text { Knowledge Project: } \\
\text { http://pkp.sfu.ca }\end{array}$ \\
\hline
\end{tabular}

\section{Gerincia da Revista} Configura a revista; cadastra editores, avaliadores, editores de texto, leitores de prova

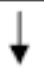

Escolhe Avalladores Editor solicta avaliação de acordo com interesses e carga

Realizaçäo de Avaliação Avaliador encaminha avaliação cega $e$ recomendações (podendo ser classificadas pelo editor)

3. Ediçilo da Submisstio

a) Edição de texto

b) Criação das composições

c) Leitura de provas

Ediçāo de Texto Editor de Layout prepara composições em HTML,PDF, PS, etc.

4. Gerência da Edição

a) Cria edições

b) Agenda submissões

c) Organiza Sumário

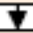

5. Publicaçäo

Oferece acesso livre imediato ou adiado, com controle de assinaturas
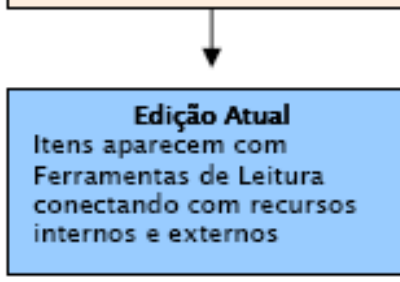

Arqulvo da Rovista Acesso a ediçöes, com indexação por sistemas $\mathrm{OAl}$. bem como Google, etc. 
A revista oferece acesso livre e imediato ao seu conteúdo, seguindo o princípio de que disponibilizar gratuitamente o conhecimento científico ao público proporciona de fato a democratização mundial do conhecimento. Por sua vez, vale ressaltar que a PG\&C não possui financiamento de qualquer tipo. Todo o trabalho editorial e de avaliação é realizado de modo voluntário. Dessa forma, a equipe editorial da PG\&C se vale de um trabalho em rede compartilhado e colaborativo. Diferentemente de algumas revistas científicas, a PG\&C não possui infraestrutura física institucional (salas, telefones, computadores, etc.). No entanto, com a classificação no sistema Qualis - o que é sempre uma exigência - buscar-se-á financiamento por meio da elaboração de projeto para submissão a editais públicos de apoio às revistas científicas.

Acerca da demanda de submissões à revista, entendida aqui como resultado do processo editorial, desde o mês de dezembro de 2010 (quando da disponibilização online do sistema da revista), foram recebidas 327 submissões de artigos de autores nacionais e estrangeiros.

Das submissões, em observância ao processo de dupla avaliação por pares, 122 foram aprovadas e publicadas, 110 foram rejeitadas e arquivadas, 94 submissões foram contabilizadas como inadequadas por não se encaixarem no foco e escopo da revista e, desde sua criação, apenas uma submissão foi arquivada por solicitação dos autores.

Ademais, além das 327 submissões de artigos, foram publicados na PG\&C outros 24 documentos referentes a 9 editoriais, 1 apresentação de seção, 3 resenhas, 3 pontos de vista e 9 expedientes.

Sobre o número de submissões rejeitadas e arquivadas, ressaltamos que tal situação não surpreendeu a Equipe Editorial. Entendemos que este número atesta o necessário rigor dos avaliadores quanto ao enquadramento das submissões ao foco, escopo e normas da revista, além da evolução contemporânea do status quo práticoepistemológico da relação entre as temáticas Gestão e Conhecimento, em prol da qualidade da publicação, ainda mais quando do lançamento de uma nova revista.

\subsection{Principais assuntos dos artigos publicados}

Para a contabilização dos principais assuntos dos artigos publicados, considerou-se para tal os artigos de revisão, os relatos de pesquisa, os relatos de experiência e os artigos que compuseram a seção memória de eventos científico-profissionais.

Neste sentido, dos 122 artigos publicados e considerados na pesquisa, 307 assuntos foram citados como palavraschave no momento de submissão de originais através do sistema do periódico. Considerando a profusão de assuntos na relação entre Gestão e Conhecimento, 42 termos se repetiram - como indicado na Tabela 1, seguido do número de freqüência com que ocorreram.

Tabela 1 : Assuntos mais frequentes na PG\&C identificados mediante palavras-chave dos artigos (continua)

\begin{tabular}{l|c}
\hline \multicolumn{1}{c|c}{ ASSUNTOS } & Frequência \\
\hline Gestão do Conhecimento & 29 \\
\hline Gestão da informação & 12 \\
\hline Ciência da Informação & 10 \\
\hline Aprendizagem & 8 \\
\hline Sistemas de Informação & \\
\hline Tecnologias da Informação & 7 \\
\hline Comportamento & 6 \\
\hline Compartilhamento da informação e do conhecimento & \\
\hline Ética & 5 \\
\hline Arquivística & \\
\hline Redes Sociais & \\
\hline Competências & \\
\hline Arquitetura da Informação & \\
\hline Inteligência & \\
\hline Informação & \\
\hline Capital Intelectual & \\
\hline Fontes de informação & \\
\hline
\end{tabular}

Fonte: Autoria própria (2013) 
Tabela 1 : Assuntos mais frequentes na PG\&C identificados mediante palavras-chave dos artigos (conclusão)

\begin{tabular}{|c|c|}
\hline $\begin{array}{ll} & \text { ASSUNTOS } \\
\text { Conhecimento } & \end{array}$ & \multirow[t]{11}{*}{ 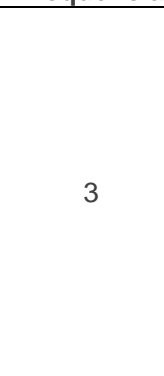 } \\
\hline $\begin{array}{l}\text { Conhecimento } \\
\text { Conhecimento Tácito }\end{array}$ & \\
\hline Conhecimento Explícito & \\
\hline Gestão & \\
\hline Gestão de pessoas & \\
\hline Gestão da informação e do conhecimento & \\
\hline Gestão eletrônica de documentos & \\
\hline Comunicação & \\
\hline Hermenêutica & \\
\hline Responsabilidade Sócio-ambiental & \\
\hline Software & \\
\hline Universidade Federal da Bahia & \multirow{13}{*}{2} \\
\hline Cognição & \\
\hline Território & \\
\hline Necessidade Informacional & \\
\hline Usabilidade & \\
\hline Estrutura Organizacional & \\
\hline Estudo de usuários & \\
\hline Usuários da informação & \\
\hline Responsabilidade social & \\
\hline Ética da informação & \\
\hline Revistas científicas & \\
\hline Semiótica & \\
\hline Treinamento e Desenvolvimento & \\
\hline
\end{tabular}

Fonte: Autoria própria (2013)

Entre os termos que refletem os assuntos, destacaram-se pela frequência com que foram adotados pelos autores, os termos Gestão do conhecimento, Gestão da Informação, Ciência da Informação, Aprendizagem, Sistemas e Tecnologias de informação. Também aparecem com freqüente ocorrência os termos Comportamento e Compartilhamento da informação e do conhecimento, seguidos de Ética, Tomada de decisão, Competências, Arquivística e Redes Sociais. Destarte, os resultados obtidos expressam a pertinência às tendências dos estudos convergentes entre a Ciência Administrativa e a Ciência da Informação, além da relação com áreas afins. Este espectro de assuntos demonstra, ainda, a pluralidade de práticas e saberes que refletem as temáticas foco da PG\&C, validando a necessidade de sua existência e manutenção.

\section{Considerações finais}

O projeto "Perspectivas em Gestão \& Conhecimento" se formou em face da idealização compartilhada da revista, como produto cooperativo de trabalho exaustivo e generoso de muitos na sua idealização.

Desde a sua idealização até a sua evolução como canal de comunicação científica, tem-se que a "doença dos três números" que expressa um sério problema enfrentado por incontáveis revistas científicas em que ocorre o atraso em suas publicações ou pior ainda o desaparecimento primário de revistas recém-criadas (SOUZA, 1992) não acometeu a PG\&C, haja vista a regularidade de suas edições pelo cumprimento rigoroso de sua periodicidade, além do lançamento de um número especial por ano.

O reconhecimento e a contribuição da revista para as áreas da Ciência da Informação, da Administração e outras áreas afins, na promoção de diálogos necessários e atuais, são constatados na sua indexação nacional e internacional, no acesso da publicação, na demanda de submissões, na qualidade das avaliações e dos itens publicados, além da classificação no sistema Qualis voltado à pós-graduação stricto sensu brasileira. Pontua-se, assim, o acompanhamento por parte da Equipe Editorial do "desenvolvimento e dos critérios exigidos para a manutenção da qualidade de uma revista científica (GARCIA, 2006, p. 170). 
É fato que um periódico devidamente estruturado em termos de conteúdo e formatação promove a recuperação de itens de interesse de forma otimizada, promovendo, ainda, o reconhecimento de produção científica de autores, e o trabalho de consultores, avaliadores e editores.

\section{Referências}

BARDIN, L. (2008). Análise de conteúdo. 7. ed. Lisboa: Edições 70, LDA.

GARCIA, J. C. R. (2006). Desafios das publicações científicas. Intercom: Revista Brasileira de Ciências da Comunicação, São Paulo, v.29, n.1, p. 169-173, jan./jun. 2006.

GOMES, J. O.; COSTA, L. F. (2011). Bem-aventurança à multiplicação e ao encontro de olhares e saberes sobre Gestão e Conhecimento. Perspectivas em Gestão \& Conhecimento, v. 1, n. 1, p. 1-3.

OLIVEIRA, J. F. C.; PINHEIRO, L. V. R; ANDRADE, A. R. (2011). Informação como objeto para construção do corpus interdisciplinar entre Ciência da Informação e Ciência da Administração. Liinc em Revista, v.7, n.1, mar., p.61-81.

PUBLIC KNOWLEDGE PROJECT (PKP); INSTITUTO BRASILEIRO DE INFORMAÇÃO EM CIÊNCIA E TECNOLOGIA (IBICT) (2009). OJS em uma hora. Uma Introdução ao Sistema Eletrônico de Editoração de Revistas SEER/OJS Versão 2.1.1. Disponível em: <http://www.ibict.br/anexos secoes/OJSinanHour2.1.1.pt br.vrs1.0.pdf>. Acesso em: 10 jun 2009.

PERSPECTIVAS EM GESTÃO \& CONHECIMENTO (PG\&C) (2013a). Disponível em: <http://periodicos.ufpb.br/ojs2/index.php/pgc/index>. Acesso em: 12 mar. 2013.

(2013b). Declaração de ética e boas práticas de publicação. Disponível em: <http://periodicos. ufpb.br/ojs2/index.php/pgc/about/editorialPolicies\#custom-2>. Acesso em: 12 mar. 2013.

(2013c). Indexação. Disponível em: <http://periodicos.ufpb.br/ojs2/index.php/pgc/about/editorialPolicies\#custom-3>. Acesso em: 12 mar. 2013.

RICHARDSON, R. J (1999). Pesquisa social: método e técnicas. 3. ed. São Paulo: Atlas.

SILVA, A. C. P.; COSTA, L. F (2010). O acesso livre aos periódicos científicos eletrônicos em Turismo no Brasil. In: RAMOS, S. P.; MUNDET-I-CERDÁN, L. Turismo, Políticas e Desenvolvimento Humano. Porto Alegre: Asterisco, p. 131-164.

SOUZA, D. H. F. (1992). Publicações periódicas: processos técnicos, circulação e disseminação seletiva da informação. Belém: Universidade Federal do Pará.

\footnotetext{
${ }^{1} \mathrm{Na}$ data de 17 de fevereiro de 2014 foram registrados 50.213 acessos desde 91 países. A localização de cada visita é baseada no endereço IP do computador usado, sendo um endereço IP por visita em um período de 24 horas. Ver mapa de acessos em: http://www2.clustrmaps.com/pt/counter/maps.php?url=http://periodicos.ufpb.br/ojs2/index.php/pgc\#totals.

${ }^{2}$ Software criado pela equipe LOCKSS da Universidade de Stanford, originalmente desenvolvido para a preservação de periódicos eletrônicos.

${ }^{3}$ Este sistema classifica periódicos citados anualmente pelos programas de pós-graduação stricto sensu do Brasil, conforme citação no programa Coleta CAPES, para composição das Avaliações Trienais de Áreas de Conhecimento. A classificação gerada está disposta por áreas do conhecimento em estratos que vão de A1 (periódico de maior qualidade), A2, B1, B2, B3, B4, B5 e C (não periódicos, publicações inadequadas, com peso zero). Ver: http://www.capes.gov.br/avaliacao/qualis.
} 


\section{Dados dos autores}

\section{Luciana Ferreira da Costa}

Professora do Departamento de Ciência da Informação da Universidade Federal da Paraíba. Doutoranda em História e Filosofia da Ciência com Especialidade em Museologia pela Universidade de Évora. Mestre em Ciência da Informação pela Universidade Federal da Paraíba.

lucianna,costa@yahoo.com.br

\section{Emeide Nóbrega Duarte}

Professora do Departamento de Ciência da Informação da Universidade Federal da Paraíba. Doutora em Administração pela Universidade Federal da Paraíba.

emeide@hotmail.com

\section{Jorge de Oliveira Gomes}

Professor do Departamento de Administração da Universidade Federal da Paraíba. Mestre em Informática pela Universidade Federal de Campina Grande (UFCG)

prof.jorgeogomes@gmail.com

\section{Alan Curcino Pedreira da Silva}

Professor da Universidade Federal de Alagoas. Doutorando pelo Programa Integrado de Doutorado em Filosofia das Universidades Federais da Paraíba, Pernambuco e Rio Grande do Norte. Mestre em Ciência da Informação pela Universidade Federal da Paraíba

alancurcino@hotmail.com

Recebido - Received : 2014-02-20

Aceitado - Accepted : 2014-09-30 
Biblios A revista Perspectivas em Gestão \& Conhecimento (PG\&C)

\section{(c) $\mathbf{E Y}$}

This work is licensed under a Creative Commons Attribution 4.0 United States License.

\section{ULIS D-Sont}

This journal is published by the University Library System of the University of Pittsburgh as part of its D-Scribe Digital Publishing Program and is cosponsored by the University of Pittsburgh Press. 